\title{
Ambulatory Microdose PET: A Wearable PET Scanner for Neurologic Imaging
}

\author{
Rani M. Noble \\ Nuclear Medicine Technology Program, Gateway Community College, Phoenix, Arizona
}

\begin{abstract}
The ambulatory microdose PET scanner is an innovative machine that is advancing the field of nuclear medicine neurologic imaging. This scanner is a wearable helmet that allows neurologic PET imaging to be done while a subject is sitting upright or ambulating. It has the potential to tell researchers and physicians more about how the brain and movement are connected and give more insight on the deeper structures. The evolving design of the helmet has grown to show improvement in image quality and the ability to image the brain with useful information on different neurologic disorders and diseases. When the ambulatory microdose PET scanner is compared with a conventional PET scanner, it not only provides higherresolution images and increased sensitivity while allowing the patient to have a greater range of motion but also allows for the introduction of newer radiotracers and lower radiation doses.
\end{abstract}

Key Words: brain; wearable; positron emission tomography; microdose

J Nucl Med Technol 2019; 47:336-340

DOI: 10.2967/jnmt.119.228718

$\mathbf{T}$ here have been significant advances in medical imaging that have helped answer fundamental questions about the human body. However, it seems these advances demonstrate only how little is known. Neurologic imaging is one of the most critical areas of medicine being investigated because a complete understanding of the functioning of the brain is still lacking.

One crucial area for investigation is how the brain functions while an individual naturalistically responds to everyday challenges. Information such as this will provide insight into how movement and the brain are connected. To figure this out, an innovative neurologic imaging prototype, the ambulatory microdose (AM) PET machine, has recently been developed. In the future, knowledge gained from AM PET may lead to better treatment and management of certain neurologic conditions and diseases.

Received Mar. 14, 2019; revision accepted Apr. 3, 2019.

For correspondence or reprints contact: Rani M. Noble, Gateway Community College, 108 40th St., Phoenix, AZ 85034.

E-mail: raninoble@yahoo.com

Published online Jun. 10, 2019.

COPYRIGHT (C) 2019 by the Society of Nuclear Medicine and Molecular Imaging.
The results of AM PET will allow for neurologic imaging to be accomplished while a patient performs actions, such as walking. AM PET is the future of brain research and uses state-of-the-art technology to obtain images. This novel technology not only will help researchers get clearer images of parts of the brain that are associated with movement but also will pave a path toward enhancing the precision of nuclear imaging and lowering radiation doses to patients.

\section{THE ORIGIN OF THE WEARABLE PET SCANNER}

The idea for a wearable PET scanner for neurologic imaging began with the rat conscious animal PET (RatCAP) device. The RatCAP allowed researchers to image the brains of the rats while they were awake as opposed to being anesthetized, dramatically affecting the outcome of the results (6).

The RatCAP inventors modeled the scanner after conventional PET scanners; however, they had to miniaturize everything by using 12 mini detector blocks in a ring. The scanner weighs only $250 \mathrm{~g}(\sim 0.5 \mathrm{lb})(1)$, allowing researchers to image the brain while the rat moves about a $40 \times 40 \mathrm{~cm}$ test chamber with the device directly attached to its head (2). The success of these preclinical studies of imaging in real time on moving animals marked the beginning of an ingenious idea that could change the future of neurologic imaging in humans.

\section{WEARABLE PET SCANNER TECHNOLOGY FOR HUMANS}

To create the AM PET for human use, Julie BrefczynskiLewis, a neuroscientist, and Stan Majewski, a physicist, from the West Virginia University had to find a way to configure a full-sized PET scanner into a ring that could fit around the head of an averaged-sized person (1). Conventional PET scanners commonly use larger-sized photomultiplier tubes. However, silicon photomultipliers, which are now readily available, were incorporated into the PET ring with the support of an acrylic frame (1). The ring itself includes 12 photodetector modules, allowing the model to be more compact yet still have high image resolution due to the new silicon photomultipliers (3). The small size of the silicon photomultipliers allows the ring to be positioned very close to the head, resulting in an increase in sensitivity of more than $400 \%$ compared with traditional PET 
scans (3). The vertical field of view is $5 \mathrm{~cm}$, and the imaging interval is $30 \mathrm{~s}$. The ring also has 10 -mm-thick lutetiumyttrium oxyorthosilicate crystal scintillators (1). This first AM PET model was called generation 0 (Fig. 1).

The original design for the human-sized AM PET weighed $2.4 \mathrm{~kg}(5.28 \mathrm{lb})(1)$. This weight posed several obstacles related to supporting the helmet. When the wearer limited head motion, the helmet was steady, but if the wearer moved more vigorously, motion artifacts resulted. Other obstacles included coregistration issues originating from the tight fit around the wearer's head, giving rise to blurry images. Heat arising from the photodetectors and the wearer's head increased image noise. Multiple helmet positions were also needed to obtain full images of the brain (1). Even though the generation 0 device showed promising results, with elevated uptake in important structures within the brain (Fig. 2 ), these problems indicated the need for an improved prototype, generation 1 .

\section{UNWEIGHTING SYSTEM}

The specific goal of the generation 1 machine was to create high-definition images while allowing the wearer to have a greater range of motion. The inventors achieved this goal through better support of the helmet weight. Instead of an acrylic structure, the inventors used a 3-dimensionally printed helmet with a 4-tiered ring to image the entire brain with 1 helmet position (Fig. 3) (1). The revised prototype also included a new Biodex Unweighting System, a supporting system originally used in physical therapy to help patients with difficulty walking (Fig. 4). The unweighting system helps to support the helmet comfortably and safely by its counterweight-and-pulley system (1). A ball-andsocket joint is used to allow for head rotation in all directions (1). The system is also connected to a track that can support up to $136 \mathrm{~kg}$ ( $\sim 300 \mathrm{lb})$, accommodating a large

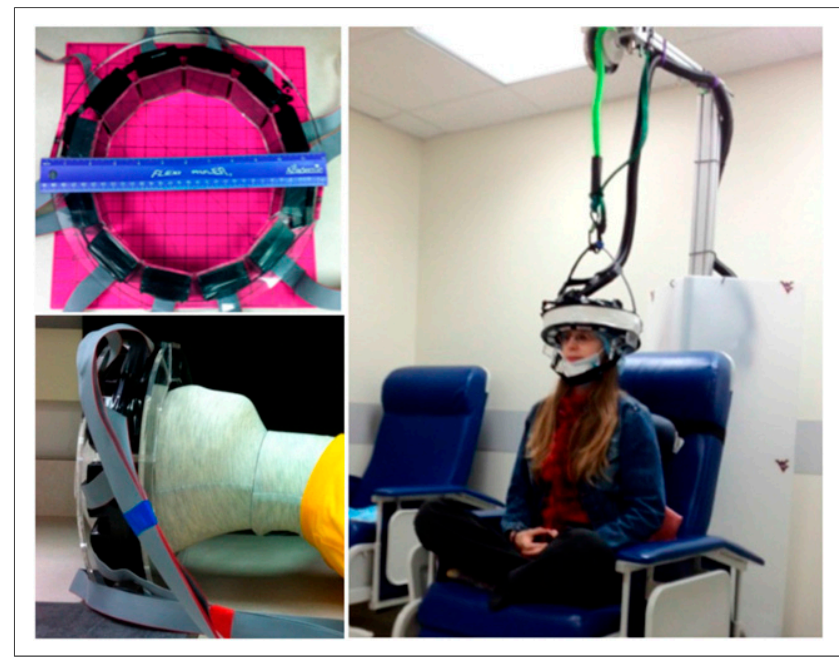

FIGURE 1. (Left) Original (generation 0) AM PET design. (Right) AM PET original bungee cord support frame. (Reprinted with permission of (6).)

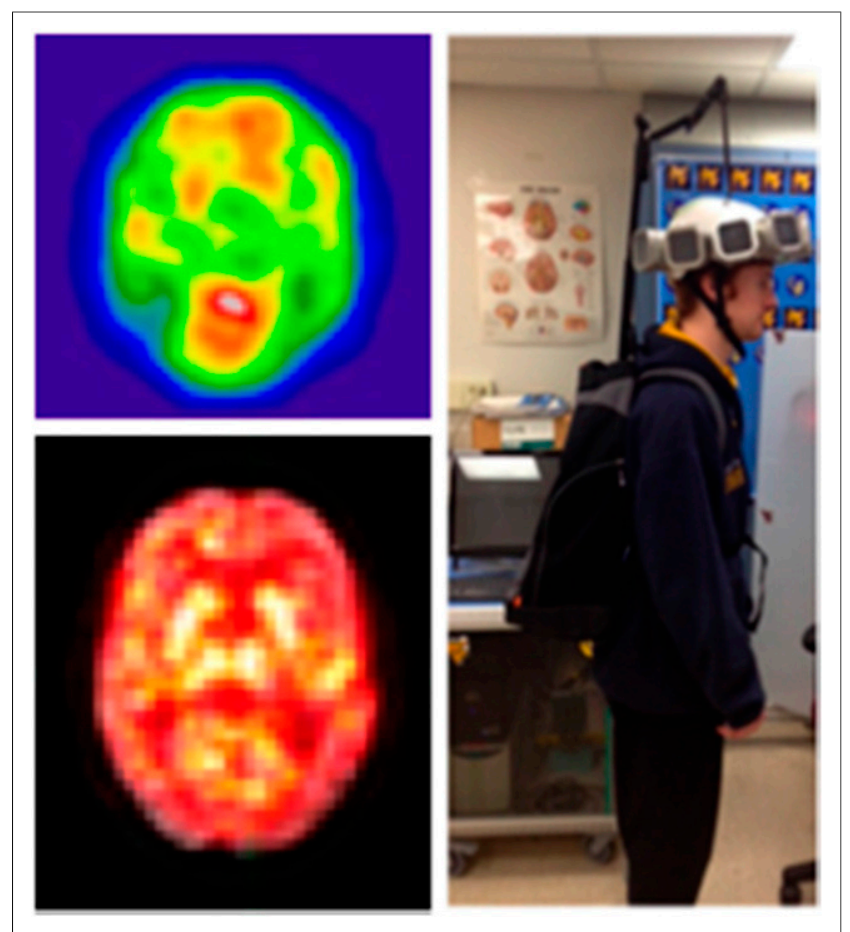

FIGURE 2. Generation 0 images. (Top left) A 2-mm-thick slice of brain after 1-min acquisition during head movement. A 333to $444-\mathrm{MBq}(9-12 \mathrm{mCi})$ dose of ${ }^{18} \mathrm{~F}-\mathrm{FDG}$ was injected, and imaging took place after 2 half-lives had passed $(74-111 \mathrm{MBq}$, or $2-3 \mathrm{mCi})$. Uptake is shown in frontal lobes and occipital cortex. Head of caudate can also be clearly identified. (Bottom left) Separate 2-mm slices were overlaid onto PET scan. This image was taken over 10 min. (Right) Concept of mobile imaging, using backpack to store electronics and support system for helmet. (Reprinted with permission of (6).)

range of patients. This configuration enables the patient to walk on a treadmill while being imaged (1).

\section{UNWEIGHTING SYSTEM TESTING RESULTS}

The inventors first tested the unweighting system during movement using the generation 1 AM PET helmet.

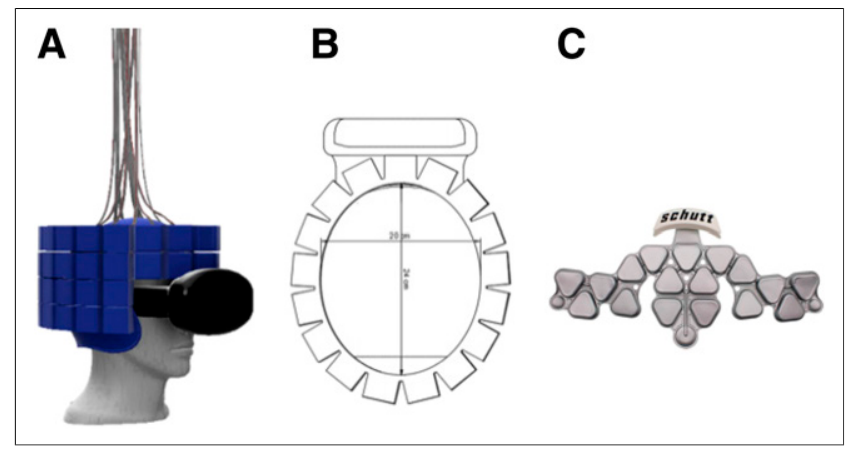

FIGURE 3. (A) Side view of 4-tiered 3-dimensionally printed helmet with virtual reality set. (B) Top view of 4-tiered helmet design. (C) Air bladder used for concept in 3-dimensionally printed helmet used to help give helmet tight and comfortable fit. (Reprinted with permission of (6).) 


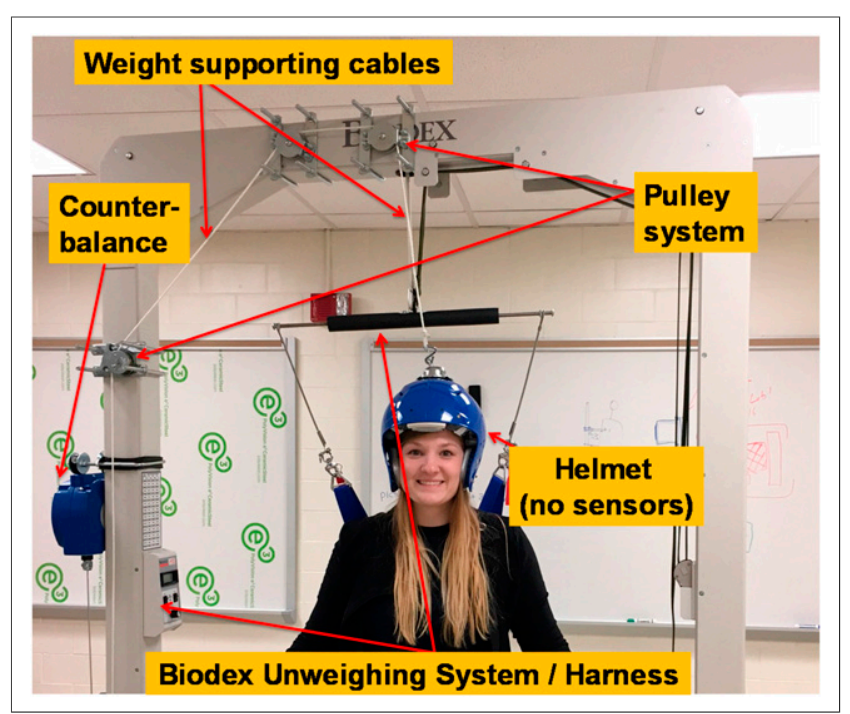

FIGURE 4. Generation 1 support design, showing counterbalance that can support up to $10 \mathrm{~kg}$ but has ability to be changed. (Reprinted with permission of (6).)

Participants alternated between moving their finger and tapping their foot in six 1-min cycles of $30 \mathrm{~s}$ per movement. The data revealed that each movement had a unique brain activation that was attributed to specific brain motor network regions.

Next, the investigators scanned 2 patients alternating between walking and standing. One of the patients had the helmet placed superiorly on the head, and the other had it placed inferiorly on the head. The goal was to image different parts of the brain and compare uptake location. The first patient, wearing the helmet higher on the head, had uptake in the motor cortex, which is associated with the leg region. The second patient, wearing the helmet lower on the head, confirmed basal ganglia uptake, which is associated with motor control, emotions, behavior, and motor learning $(1,4)$.

\section{NEUROLOGIC IMAGING POSITIONING SYSTEMS}

Generation 1 can be used with various support systems to allow imaging while the patient is sitting, standing, and possibly ambulatory. Each option for support has its own advantages and disadvantages (Table 1) $(1,5)$.

Use of AM PET on a seated patient allows the helmet to be supported from above and enables free motion of the head and upper body of the wearer; however, this type of scanning position limits leg movement. It provides the most support for the helmet, resulting in higher sensitivity, resolution, and coverage. This position is more likely to be used for research on Alzheimer disease, dementia, memory, epilepsy, navigation, social interactions, and emotions, along with attention deficit hyperactivity disorder and addiction research (6).

Another AM PET support system is called exostructure support (6). This system permits patients to stand during scanning while the helmet is supported by an overhead track as previously described. This type of support lets patients move their head and upper body while walking and balancing. However, patient imaging is restricted to the laboratory. Since most of the weight of the helmet is supported by the overhead track, this support system provides comparatively high sensitivity, resolution, and coverage. The higher sensitivity results from the helmet's weight being supported by another object, which also allows the helmet to be more steady and to move less. This option will be useful in research on Parkinson disease, traumatic brain injuries, social interactions, stroke, and physical therapy (6).

The last alternative for a positioning system allow the most movement. The support for the helmet is built into a backpack that enables the patient to leave the laboratory and have the most head movement. Because the weight of the helmet does not have as much support with this system, it has a noticeably reduced sensitivity and resolution. The patient is required to support 10 pounds of extra weight. This configuration will be useful for natural-behaviors imaging and research (6).

\section{RADIOTRACERS}

Conventional PET scans are typically done using the radiotracer ${ }^{18} \mathrm{~F}$-FDG to show metabolic changes. With the

TABLE 1

Traditional PET Scanners Compared with Upright PET Scanners

\begin{tabular}{|c|c|c|c|c|c|c|c|}
\hline Scanner & General feature & Geometry & Motion-friendly & $\begin{array}{l}\text { Spatial } \\
\text { resolution }\end{array}$ & Sensitivity (\%) & Axial coverage & Support mechanics \\
\hline $\begin{array}{l}\text { Whole-body } \\
\text { PET }\end{array}$ & Standard of care & $\begin{array}{l}\text { Horizontal, supine, } \\
\text { or prone }\end{array}$ & No & $4-5 \mathrm{~mm}$ & 1 & Whole brain & Bed \\
\hline Helmet PET & $\begin{array}{l}\text { Wearable feasibility } \\
\text { prototype }\end{array}$ & Upright & Yes & $2-4 \mathrm{~mm}$ & 0.5 & $\sim 5 \mathrm{~cm}$ & $\begin{array}{l}\text { Passive vertical } \\
\text { weight support }\end{array}$ \\
\hline $\begin{array}{l}\text { AM PET, } \\
\text { generation } 0\end{array}$ & $\begin{array}{c}\text { Dissemination } \\
\text { prototype }\end{array}$ & Upright & Yes & $2-5 \mathrm{~mm}$ & $3-5$ & Whole brain & $\begin{array}{l}\text { Passive weight } \\
\text { support }\end{array}$ \\
\hline $\begin{array}{l}\text { AM PET, } \\
\text { generation } 1\end{array}$ & $\begin{array}{l}\text { Dissemination } \\
\text { prototype }\end{array}$ & Upright & Yes & $2-3 \mathrm{~mm}$ & $5-10$ & Whole brain & $\begin{array}{l}\text { Active weight } \\
\text { support }\end{array}$ \\
\hline
\end{tabular}


AM PET wearable scanner and the updated technology, it is possible to use alternative radiotracers that show activity related to specific neurotransmitter systems (7). For example, radiolabeled ligand targets are being investigated as a noninvasive way to show these specific systems, pathways, and neuroreceptors within the brain. Ligands are preferred to enzymes because of the higher rate of accumulation that is measurable within the time limit of PET imaging. Examples include the $\mathrm{D}_{2}$ dopamine receptor antagonist ${ }^{11} \mathrm{C}$-raclopride, ${ }^{11} \mathrm{C}-\mathrm{MADAM}$, and ${ }^{11} \mathrm{C}$-ZIENT for serotonin transporter binding, as well as other opioid ligands (7).

In addition to alternative radiotracers being researched, radiation doses to patients might be lowered by the AM PET. This is due to the components of the helmet that allow for the scanner to be nearer the head, combined with photomultipliers that detect positron events with greater efficiency. Early acquisitions with the helmet produced quality images at $25 \%$ of the injected dose. The creators predict that high-quality images may be obtained with only $10 \%$ of a standard dose (l).

\section{EARLY STUDY RESULTS}

In a 2016 study, investigators used the AM PET scanner to image 4 men with known cancer. At the facility managing their care, the usual treatment protocol required a brain PET/CT follow-up to a full-body PET/CT scan. Instead of undergoing the head scan on a traditional PET/ CT machine, the patients were scanned using AM PET. Two of the patients were scanned immediately after their full-body PET/CT scan, and the other two were imaged $4 \mathrm{~h}$ after injection (7).

The researchers acquired images in 4 sequences. The first was a fast 30-s scan of the brain, the second was a 3-min scan, the third was a 10-min scan, and the fourth was a 1-min scan. During the 1-min scan, the patient turned his head left and right about $45^{\circ}$ in each direction. After the image acquisition, regions of interest were drawn over specific regions of the brain to calculate uptake (7).

The images of the 2 participants scanned immediately on completion of full-body PET/CT were overly saturated: there was too much activity, resulting in poor image quality. The 2 patients imaged $4 \mathrm{~h}$ after injection, allowing for radioactive and biologic decay of the tracer, had usable images. These results suggest that lower radiation doses used with AM PET will produce better-quality images (7).

When the investigators compared the AM PET images acquired during deliberate head movement with standard PET/CT brain images, the results revealed prevalent uptake in the thalamus, basal ganglia, and occipital lobe, demonstrating the utility of the wearable PET scanner on humans in motion (7).

\section{OBSTACLES}

Even though the AM PET device has numerous superior qualities and advantages over conventional PET machines, it still has to overcome a few hurdles. The structure and design are still under development to decrease weight, enhance heat dissipation, and improve fit. Another area under consideration is the feasibility of a pediatric model that can accommodate the varying head sizes of children.

The inventors' primary goal for AM PET is to be able to image patients during movement. Unfortunately, even with the advances in helmet design, the speed and variety of possible movement continue to be limited because of the equipment weight. Related to the weight problem is the need for shielding to reduce scatter from the rest of the body. Shielding, unfortunately, further increases the weight (7).

As yet, investigators have completed few human studies using the AM PET device. Most studies have been conducted on rats or to further improve the design of the helmet in humans. At this writing, the AM PET device is beginning clinical research trials, but it will be many years before the device is approved and available for widespread use.

\section{CONCLUSION}

To help researchers improve the field of neurologic imaging, AM PET seems to be the answer to many issues arising from conventional scanner use. Additionally, when compared with traditional PET scanning, AM PET scanning provides higher-resolution images due to the proximity of the detector to the brain, delivers a lower radiation dose to the patient, and will be motion-tolerant (6). A lower radiation dose to the patient is important in allowing for more frequent scans, which will, in turn, mean better disease management. Physicians will be able to better understand how a patient is responding to treatment and change the course of treatment more quickly if needed.

In the past, it has been difficult to obtain quality images distinguishing deep brain structures. However, it may now be possible to gather better information on these areas through the use of AM PET, which is positioned closer to the brain and uses newer technology and newer radioisotopes. In conjunction with the new radiotracers that are becoming available, researchers can ask patients to perform a specific movement or task and interpret how that action changes the uptake in the brain. In a conventional PET machine, new radiotracers could be used but movements such as this are not possible because of scanner design constraints.

Lastly, AM PET has the possibility of improving imaging during movement and aiding in the diagnosis and treatment of patients with various neurologic ailments such as Alzheimer disease, Parkinson disease, dementia, stroke, attention deficit hyperactivity disorder, and brain trauma. Moreover, AM PET can benefit the pediatric population, and patients might be imaged without sedation. There are so many possible benefits to the use of AM PET that the few that have been mentioned here are only the beginning of what can be imagined.

\section{DISCLOSURE}

No potential conflict of interest relevant to this article was reported. 


\section{REFERENCES}

1. Melroy S, Bauer C, Mchugh M, et al. Development and design of next-generation head-mounted ambulatory microdose positron-emission tomography (AM-PET) system. Sensors (Basel). 2017;17:1164.

2. Vaska P, Woody CL, Schlyer DJ, et al. RatCAP: miniaturized head-mounted PET for conscious rodent brain imaging. IEEE Trans Nucl Sci. 2004;51:2718-2722.

3. Freeman T. Portable brain scanner allows PET in motion. Physics World website. https://physicsworld.com/a/portable-brain-scanner-allows-pet-in-motion/. Published November 2, 2015. Accessed August 8, 2019.
4. Lanciego JL, Luquin N, Obeso JA. Functional neuroanatomy of the basal ganglia. Cold Spring Harb Perspect Med. 2012;2:a009621.

5. Majewski S, Proffitt J, inventors. Jefferson Science Associates LLC, assignee. Compact and mobile high resolution PET brain imager. U.S. patent 7,884,331. February $8,2011$.

6. About the AM-PET. AM-PET imaging website. https://www.pethelmet.org/. Published 2015.

7. Bauer CE, Brefczynski-Lewis J, Marano G, et al. Concept of an upright wearable positron emission tomography imager in humans. Brain Behav. 2016;6: e00530. 